SUPPORTING INFORMATION

\title{
MULTICENTER BOND INDICES AS A NEW MEANS FOR THE QUANTITATIVE CHARACTERIZATION OF HOMOAROMATICITY.
}

Robert Ponec, Patrick Bultinck, Ana Gallegos Saliner

The following data are included in the supporting information :
A. Computational parameters
B. Energy, number of imaginary frequencies and Cartesian coordinates for all compounds

A. Computational methods and input parameters :

- B3LYP functional as implemented in Gaussian-03. 6-31G* basis set, using cartesian $\mathrm{d}$ and $\mathrm{f}$ basis functions $(6 \mathrm{D}, 10 \mathrm{~F}$ keywords).

- NICS calculated as the negative of isotropic shifts calculated via inclusion of a ghost atom $(\mathrm{Bq})$ at ring centers of the hypothetical ring formed by the five atoms in the 5-center bond (Keyword NMR). 
B. Energy, number of imaginary frequencies and Cartesian coordinates for all compounds :

Reported energies, NICS and optimized geometries refer to B3LYP/6-31G*// B3LYP/6-31G* calculations. Cartesian coordinates in $\AA$. All structures were identified with zero imaginary frequencies.

- Series I, X=BH

Energy $=-296.959003227$ Hartree

$\mathrm{NICS}=3.5054$

5 -center index $=-0.00277$

Cartesian coordinates (atom number, $\mathrm{X}, \mathrm{Y}, \mathrm{Z}$ )

$\begin{array}{lrrrr}6 & & 0.701354 & 1.077811 & 0.423272 \\ 6 & & 1.398819 & 0.405615 & -0.770985 \\ 6 & 1.048156 & -0.880205 & -0.847753 \\ 6 & & 0.053357 & -1.211437 & 0.281021 \\ 6 & & 0.501497 & -0.161692 & 1.335631 \\ 6 & & -1.377718 & -0.887668 & -0.127567 \\ 6 & & -1.769676 & 0.392700 & -0.315393 \\ 5 & & -0.712659 & 1.518968 & -0.158642 \\ 1 & & 1.296350 & 1.880834 & 0.870606 \\ 1 & & 2.033410 & 0.942062 & -1.469448 \\ 1 & & 1.348766 & -1.595427 & -1.606451 \\ 1 & & 0.131005 & -2.246239 & 0.630547 \\ 1 & & 1.450973 & -0.466338 & 1.793505 \\ 1 & & -0.244112 & -0.018635 & 2.125158 \\ 1 & & -2.061876 & -1.725304 & -0.275701 \\ 1 & & -2.797249 & 0.580525 & -0.627385 \\ 1 & & -0.928706 & 2.642945 & -0.516985\end{array}$


- Series $\mathrm{I}, \mathrm{X}=\mathrm{AlH}$

Energy $=-514.538870432$ Hartree

$\mathrm{NICS}=0.5802$

5 -center index $=-0.00546$

Cartesian coordinates (atom number, $\mathrm{X}, \mathrm{Y}, \mathrm{Z}$ )

$\begin{array}{cccc}6 & -0.847589 & -1.012513 & 0.661890 \\ 6 & -1.304897 & -0.773743 & -0.760401 \\ 6 & -1.102188 & 0.505551 & -1.133671 \\ 6 & -0.536250 & 1.331784 & 0.026457 \\ 6 & -0.932504 & 0.423490 & 1.239530 \\ 6 & 0.980518 & 1.523183 & -0.067295 \\ 6 & 1.841944 & 0.487597 & -0.091589 \\ 13 & 1.013237 & -1.283529 & 0.010768 \\ 1 & -1.402166 & -1.774729 & 1.212222 \\ 1 & -1.671027 & -1.560364 & -1.416648 \\ 1 & -1.265169 & 0.914011 & -2.126161 \\ 1 & -1.017837 & 2.314124 & 0.090504 \\ 1 & -1.970645 & 0.646155 & 1.525608 \\ 1 & -0.292527 & 0.603777 & 2.109156 \\ 1 & 1.329798 & 2.559418 & -0.097894 \\ 1 & 2.905236 & 0.722896 & -0.154779 \\ 1 & 1.618041 & -2.651508 & -0.531517\end{array}$

- Series I, X=Be

Energy $=-286.240583001$ Hartree

$\mathrm{NICS}=1.4907$

5 -center index $=-0.00308$

Cartesian coordinates (atom number, $\mathrm{X}, \mathrm{Y}, \mathrm{Z}$ )

\begin{tabular}{|c|c|c|c|}
\hline & -1.141934 & -1.046351 & 0.126530 \\
\hline & -1.479257 & 0.062470 & -0.843777 \\
\hline & -0.740670 & 1.164129 & -0.6337 \\
\hline & 0.234588 & 0.940269 & 0.52998 \\
\hline & -0.522216 & -0.190722 & 1.2942( \\
\hline & 1.621518 & 0.481728 & 0.03590 \\
\hline & 1.853394 & -0.738008 & -0.5036 \\
\hline & 0.373455 & -1.490290 & -0.4674 \\
\hline & -1.972359 & -1.686380 & 0.4267 \\
\hline & -2.184618 & -0.064196 & -1.6615 \\
\hline & -0.734110 & 2.058501 & -1.2488 \\
\hline & 0.357211 & 1.834804 & $1.1510^{2}$ \\
\hline & -1.334558 & 0.253368 & 1.8869 \\
\hline & 0.136339 & -0.741609 & 1.9754 \\
\hline & 2.418964 & 1.225410 & 0.13813 \\
\hline & 2.866778 & -0.959825 & -0.8304 \\
\hline
\end{tabular}


- Series I, $\mathrm{X}=\mathrm{Mg}$

Energy $=-471.552570099$ Hartree

$\mathrm{NICS}=1.5102$

5 -center inde $\mathrm{x}=-0.00249$

Cartesian coordinates (atom number, $\mathrm{X}, \mathrm{Y}, \mathrm{Z}$ )

$\begin{array}{cccc}6 & 1.396609 & 0.713835 & 0.451934 \\ 6 & 1.569727 & 0.012446 & -0.850196 \\ 6 & 0.751512 & -1.051777 & -0.988659 \\ 6 & -0.110922 & -1.257324 & 0.259115 \\ 6 & 0.675248 & -0.379816 & 1.300838 \\ 6 & -1.586112 & -0.871981 & 0.041861 \\ 6 & -2.054593 & 0.367191 & -0.216951 \\ 12 & -0.414003 & 1.627371 & -0.131275 \\ 1 & 2.288466 & 1.149027 & 0.901973 \\ 1 & 2.241326 & 0.372692 & -1.628047 \\ 1 & 0.641148 & -1.657492 & -1.882678 \\ 1 & -0.095344 & -2.307865 & 0.576272 \\ 1 & 1.434937 & -1.010568 & 1.786887 \\ 1 & 0.016247 & -0.004389 & 2.093780 \\ 1 & -2.275868 & -1.724473 & 0.099396 \\ 1 & -3.131688 & 0.459167 & -0.359924\end{array}$

- Series I, $\mathrm{X}=\mathrm{O}$

Energy $=-346.717760125$ Hartree

$\mathrm{NICS}=-1.3290$

5 -center index $=0.00548$

Cartesian coordinates (atom number, $\mathrm{X}, \mathrm{Y}, \mathrm{Z}$ )

$\begin{array}{rrrr}6 & -0.321561 & -1.152695 & 0.337782 \\ 6 & -1.224368 & -0.697007 & -0.791944 \\ 6 & -1.284304 & 0.642453 & -0.774169 \\ 6 & -0.431444 & 1.179882 & 0.376482 \\ 6 & -0.492827 & -0.016802 & 1.355984 \\ 6 & 0.997552 & 1.227101 & -0.148509 \\ 6 & 1.617492 & 0.057555 & -0.371071 \\ 8 & 1.063615 & -1.170443 & -0.142613 \\ 1 & -0.488436 & -2.162128 & 0.716824 \\ 1 & -1.612053 & -1.355804 & -1.562096 \\ 1 & -1.746545 & 1.275302 & -1.526816 \\ 1 & -0.763752 & 2.133080 & 0.796124 \\ 1 & -1.473432 & -0.095645 & 1.835772 \\ 1 & 0.290761 & 0.001416 & 2.118574 \\ 1 & 1.498343 & 2.162279 & -0.374418 \\ 1 & 2.622964 & -0.037882 & -0.770387\end{array}$


- Series I, $X=S$

Energy $=-669.700863202$ Hartree

NICS $=-2.4822$

5 -center index $=0.00512$

Cartesian coordinates (atom number, $\mathrm{X}, \mathrm{Y}, \mathrm{Z}$ )

$\begin{array}{cccc}6 & 0.029549 & -1.219931 & 0.363485 \\ 6 & -0.858773 & -1.213873 & -0.857325 \\ 6 & -1.619642 & -0.109713 & -0.860685 \\ 6 & -1.289821 & 0.756444 & 0.354452 \\ 6 & -0.747686 & -0.310780 & 1.331882 \\ 6 & -0.167260 & 1.693963 & -0.065307 \\ 6 & 1.088490 & 1.264573 & -0.271584 \\ 16 & 1.658488 & -0.402354 & -0.075034 \\ 1 & 0.296056 & -2.202348 & 0.760361 \\ 1 & -0.781681 & -1.947187 & -1.653765 \\ 1 & -2.275496 & 0.211012 & -1.664539 \\ 1 & -2.142218 & 1.313725 & 0.754311 \\ 1 & -1.579171 & -0.877650 & 1.765976 \\ 1 & -0.133749 & 0.101464 & 2.136920 \\ 1 & -0.401250 & 2.739073 & -0.256046 \\ 1 & 1.872562 & 1.935478 & -0.612193\end{array}$

- Series I, $\mathrm{X}=\mathrm{PH}$

Energy $=-613.456668329$ Hartree

$\mathrm{NICS}=-1.0464$

5 -center index $=0.00200$

Cartesian coordinates (atom number, $\mathrm{X}, \mathrm{Y}, \mathrm{Z}$ )

$\begin{array}{ccccc}6 & 0.046543 & -1.243032 & 0.351425 \\ 6 & -0.856968 & -1.227453 & -0.858161 \\ 6 & -1.639368 & -0.139884 & -0.853685 \\ 6 & -1.308241 & 0.730253 & 0.360409 \\ 6 & -0.737018 & -0.331325 & 1.329003 \\ 6 & -0.219351 & 1.706981 & -0.068774 \\ 6 & 1.046696 & 1.327667 & -0.301769 \\ 15 & 1.702685 & -0.361038 & 0.022453 \\ 1 & 0.260962 & -2.238005 & 0.753782 \\ 1 & -0.798152 & -1.967484 & -1.651282 \\ 1 & -2.313782 & 0.168788 & -1.646584 \\ 1 & -2.169407 & 1.265310 & 0.773234 \\ 1 & -1.553715 & -0.902688 & 1.785480 \\ 1 & -0.126557 & 0.103062 & 2.127912 \\ 1 & & -0.512209 & 2.742484 & -0.243161 \\ 1 & 1.775663 & 2.059031 & -0.648513 \\ 1 & 1.903160 & -0.754168 & -1.338345\end{array}$


- Series I, $X=\mathrm{NH}$

Energy $=-326.854065281$ Hartree

$\mathrm{NICS}=-2.0126$

5 -center index $=0.00816$

Cartesian coordinates (atom number, $\mathrm{X}, \mathrm{Y}, \mathrm{Z}$ )

\begin{tabular}{|c|c|c|c|}
\hline & -0.305620 & -1.173222 & 0.354568 \\
\hline & -1.186832 & -0.747223 & -0.81210 \\
\hline & -1.279933 & 0.592130 & -0.819368 \\
\hline & -0.493586 & 1.169546 & 0.356927 \\
\hline & -0.549752 & -0.018328 & 1.34631 \\
\hline & 0.946547 & 1.290433 & -0.131351 \\
\hline & 1.636428 & 0.152685 & -0.344123 \\
\hline & 1.128097 & -1.112829 & -0.03014 \\
\hline & -0.504574 & -2.168929 & 0.7616 \\
\hline & -1.526849 & -1.420308 & -1.5947 \\
\hline & -1.715144 & 1.200451 & -1.6079 \\
\hline & -0.884718 & 2.109401 & 0.75716 \\
\hline & -1.543298 & -0.118195 & 1.7945 \\
\hline & 0.207130 & 0.034933 & 2.13398 \\
\hline & 1.395572 & 2.257097 & -0.33280 \\
\hline & 2.648010 & 0.148587 & -0.74303 \\
\hline & 1.423689 & -1.849362 & -0.6628 \\
\hline
\end{tabular}

- Series II, $\mathrm{X}=\mathrm{PH}$

Energy $=-613.373429998$ Hartree

$\mathrm{NICS}=-6.7516$

5 -center index $=0.002310$

Cartesian coordinates (atom number, $\mathrm{X}, \mathrm{Y}, \mathrm{Z}$ )

\begin{tabular}{|c|c|c|c|}
\hline 5 & 0.725549 & 1.211029 & 0.346334 \\
\hline 6 & 1.369643 & 0.673898 & -0.925079 \\
\hline 6 & 1.370976 & -0.675131 & -0.922380 \\
\hline 6 & 0.727795 & -1.208678 & 0.351257 \\
\hline 6 & 0.934387 & 0.003163 & 1.300368 \\
\hline 6 & -0.747642 & -1.429001 & 0.000800 \\
\hline 15 & -1.618551 & -0.001587 & -0.113259 \\
\hline 6 & -0.750648 & 1.428147 & -0.004047 \\
\hline 1 & 1.166671 & 2.135260 & 0.733032 \\
\hline 1 & 1.608535 & 1.305871 & -1.776287 \\
\hline 1 & 1.611712 & -1.310048 & -1.770895 \\
\hline 1 & 1.170561 & -2.130494 & 0.741919 \\
\hline 1 & 1.969917 & 0.004333 & 1.655855 \\
\hline 1 & 0.259909 & 0.004463 & 2.161670 \\
\hline 1 & -1.186904 & -2.407644 & -0.138775 \\
\hline 1 & -2.905313 & -0.005007 & -0.670588 \\
\hline & $9 / 1$ & 2.406498 & -0.120 \\
\hline
\end{tabular}


- Series II, $\mathrm{X}=\mathrm{S}$

Energy $=-669.638000440$ Hartree

NICS $=-7.7620$

5 -center index $=0.03216$

Cartesian coordinates (atom number, $\mathrm{X}, \mathrm{Y}, \mathrm{Z}$ )

$\begin{array}{cccc}6 & -0.741411 & -1.198117 & 0.350417 \\ 6 & -1.306164 & -0.676012 & -0.963092 \\ 6 & -1.306342 & 0.675558 & -0.963650 \\ 6 & -0.741311 & 1.198486 & 0.349493 \\ 6 & -1.006299 & 0.000312 & 1.292021 \\ 6 & 0.732017 & 1.347707 & 0.058156 \\ 16 & 1.695189 & -0.000192 & -0.152627 \\ 6 & 0.732040 & -1.347488 & 0.058774 \\ 1 & -1.169458 & -2.139810 & 0.706956 \\ 1 & -1.485843 & -1.312507 & -1.824950 \\ 1 & -1.485261 & 1.311537 & -1.826013 \\ 1 & -1.169562 & 2.140253 & 0.705614 \\ 1 & -2.059272 & 0.000372 & 1.591070 \\ 1 & -0.376362 & 0.000763 & 2.186779 \\ 1 & 1.224704 & 2.300204 & -0.093448 \\ 1 & 1.222854 & -2.300431 & -0.096682\end{array}$

- Series II, $\mathrm{X}=\mathrm{NH}$

Energy $=-326.812364285$ Hartree

$\mathrm{NICS}=-9.6590$

5 -center index $=0.03876$

Cartesian coordinates (atom number, $\mathrm{X}, \mathrm{Y}, \mathrm{Z}$ )

\begin{tabular}{|c|c|c|c|}
\hline & 0.444515 & 1.183490 & 0.360654 \\
\hline & 1.119739 & 0.677373 & -0.905833 \\
\hline & 1.119821 & -0.677217 & -0.905887 \\
\hline & 0.444621 & -1.183513 & 0.360517 \\
\hline & 0.685104 & -0.000048 & 1.328843 \\
\hline & -1.007301 & -1.203445 & -0.037929 \\
\hline & -1.586713 & -0.000044 & -0.224256 \\
\hline & -1.007324 & 1.203413 & -0.037967 \\
\hline & 0.783184 & 2.153651 & 0.734420 \\
\hline & 1.343547 & 1.315509 & -1.756587 \\
\hline & 1.343635 & -1.315228 & -1.756735 \\
\hline & 0.783304 & -2.153695 & 0.734240 \\
\hline & 1.723639 & -0.000007 & 1.673974 \\
\hline & 0.012012 & -0.000187 & 2.192438 \\
\hline & -1.572373 & -2.081033 & -0.318060 \\
\hline & -2.532482 & -0.000073 & -0.590529 \\
\hline & $-1.3 / 22$ & 2.081051 & -0. \\
\hline
\end{tabular}


- Series II, $\mathrm{X}=\mathrm{O}$

Energy $=-346.650274918$ Hartree

$\mathrm{NICS}=-13.5152$

5 -center index $=0.04762$

Cartesian coordinates (atom number, $\mathrm{X}, \mathrm{Y}, \mathrm{Z}$ )

\begin{tabular}{|c|c|c|c|}
\hline & 0.452466 & 1.180541 & 0.363908 \\
\hline & 1.015631 & 0.681124 & -0.953114 \\
\hline 6 & 1.014697 & -0.682342 & -0.953219 \\
\hline & 0.450815 & -1.181206 & 0.363698 \\
\hline & 0.741567 & -0.000610 & 1.316693 \\
\hline & -0.993949 & -1.169546 & -0.018445 \\
\hline & -1.594018 & 0.001091 & -0.197950 \\
\hline & -0.992297 & 1.170898 & -0.018292 \\
\hline & 0.790826 & 2.159674 & 0.711104 \\
\hline & 1.166048 & 1.320277 & -1.817638 \\
\hline & 1.164186 & -1.321582 & -1.817840 \\
\hline & 0.787849 & -2.160859 & 0.710741 \\
\hline & 1.797398 & -0.001367 & 1.603910 \\
\hline & 0.117090 & -0.000253 & 2.215735 \\
\hline & -1.604007 & -2.011213 & -0.314659 \\
\hline & -1.600819 & 2.013441 & -0.315128 \\
\hline
\end{tabular}

- Series III

Energy $=-362.659587309$ Hartree

$\mathrm{NICS}=-8.6920$

5 -center index $=0.03253$

Cartesian coordinates (atom number, $\mathrm{X}, \mathrm{Y}, \mathrm{Z}$ )

\begin{tabular}{|c|c|c|c|}
\hline & 0.449739 & 1.155609 & 0.425100 \\
\hline & 1.159902 & 0.667866 & -0.834873 \\
\hline & 1.098674 & -0.683190 & -0.872016 \\
\hline & 0.329192 & -1.175841 & 0.334639 \\
\hline & 0.569415 & -0.058518 & 1.365601 \\
\hline & -1.068196 & -1.221328 & -0.143029 \\
\hline & -1.564877 & 0.042305 & -0.331632 \\
\hline & -0.947658 & 1.162484 & -0.096692 \\
\hline & 0.759896 & 2.121666 & 0.829079 \\
\hline & 1.472546 & 1.326770 & -1.639157 \\
\hline & 1.356567 & -1.311680 & -1.717960 \\
\hline & 0.560053 & -2.186742 & 0.678564 \\
\hline & 1.581171 & -0.124401 & 1.776126 \\
\hline & -0.159775 & -0.056683 & 2.181750 \\
\hline & -1.529650 & 2.031460 & -0.384692 \\
\hline
\end{tabular}


- Series IV, $\mathrm{X}=\mathrm{O}$

Energy $=-230.020576641$ Hartree

$\mathrm{NICS}=-13.1774$

5-center index $=0.05737$

Cartesian coordinates (atom number, $\mathrm{X}, \mathrm{Y}, \mathrm{Z}$ )

\begin{tabular}{|c|c|c|c|}
\hline & 0.000000 & 1.148788 & 0.000000 \\
\hline & 1.132095 & 0.393989 & 0.000000 \\
\hline & 0.697892 & -0.974455 & 0.000000 \\
\hline & -0.662317 & -0.938707 & 0.000000 \\
\hline & -1.106695 & 0.351054 & 0.000000 \\
\hline & 2.149796 & 0.759001 & 0.000000 \\
\hline & 1.319004 & -1.859420 & 0.000000 \\
\hline & -1.430558 & -1.696819 & 0.000000 \\
\hline & -0.190702 & 2.211122 & 0.000000 \\
\hline
\end{tabular}

- Series IV, $\mathrm{X}=\mathrm{S}$

Energy $=-230.020576641$ Hartree

NICS $=-13.5077$

5 -center index $=0.05846$

Cartesian coordinates (atom number, $\mathrm{X}, \mathrm{Y}, \mathrm{Z}$ )

$\begin{array}{cccc}6 & 0.011652 & -1.243607 & 0.000000 \\ 6 & 1.272581 & -0.714953 & 0.000000 \\ 6 & 1.272581 & 0.714953 & 0.000000 \\ 6 & 0.011652 & 1.243607 & 0.000000 \\ 16 & -1.199707 & 0.000000 & 0.000000 \\ 1 & 2.172780 & -1.320252 & 0.000000 \\ 1 & 2.172780 & 1.320252 & 0.000000 \\ 1 & -0.280528 & 2.285033 & 0.000000 \\ 1 & -0.280527 & -2.285033 & 0.000000\end{array}$

- Series IV, $\mathrm{X}=\mathrm{NH}$

Energy $=-230.020576641$ Hartree

NICS $=-15.6462$

5 -center index $=0.07663$

Cartesian coordinates (atom number, $\mathrm{X}, \mathrm{Y}, \mathrm{Z}$ )

\begin{tabular}{|c|c|c|c|}
\hline & 0.331564 & 1.126002 & 0.000000 \\
\hline & -0.983411 & 0.712683 & 0.000000 \\
\hline & -0.983411 & -0.712683 & 0.000000 \\
\hline & 0.331564 & -1.126002 & 0.000000 \\
\hline & 1.121910 & 0.000000 & 0.000000 \\
\hline & -1.849737 & 1.360187 & 0.000000 \\
\hline & -1.849737 & -1.360186 & 0.000000 \\
\hline & 0.769335 & -2.113852 & 0.000000 \\
\hline & 0.769335 & 2.113852 & 0.000000 \\
\hline & 2.129591 & 0.000000 & 0.000000 \\
\hline
\end{tabular}


- Series IV, $\mathrm{X}=\mathrm{PH}$

Energy $=-496.710012096$ Hartree

NICS $=-16.6072$

5 -center index $=0.08032$

Cartesian coordinates (atom number, $\mathrm{X}, \mathrm{Y}, \mathrm{Z}$ )

$\begin{array}{lllll}6 & 0.010846 & -1.312440 & 0.000000\end{array}$

$\begin{array}{llll}6 & 1.263658 & -0.710946 & 0.000000\end{array}$

$\begin{array}{llll}6 & 1.263658 & 0.710946 & 0.000000\end{array}$

$\begin{array}{llll}6 & 0.010846 & 1.312440 & 0.000000\end{array}$

$\begin{array}{lllll}15 & -1.115489 & 0.000000 & 0.000000\end{array}$

$\begin{array}{llll}1 & 2.179309 & -1.293773 & 0.000000\end{array}$

$\begin{array}{llll}1 & 2.179309 & 1.293773 & 0.000000\end{array}$

$\begin{array}{llll}1 & -0.205350 & 2.370802 & 0.000000\end{array}$

$\begin{array}{llll}1 & -0.205350 & -2.370802 & 0.000000\end{array}$

$\begin{array}{llll}1 & -2.509620 & 0.000000 & 0.000000\end{array}$ 